\title{
Supporting information Global Activity Search Uncovers Reaction Induced Concomitant Catalyst Restructuring for Alkane Dissociation on Model Pt Catalysts
}

Geng Sun ${ }^{1}$, Jack T. Fuller III, ${ }^{2}$ Anastassia N. Alexandrova ${ }^{2,3} *$, Philippe Sautet ${ }^{1,2,3 *}$

${ }^{1}$ Department of Chemical and Biomolecular Engineering, University of California, Los Angeles, Los Angeles, California 90095, United States

${ }^{2}$ Department of Chemistry and Biochemistry, University of California, Los Angeles, Los Angeles, California 90095, United States

${ }^{3}$ California Nano Systems Institute, Los Angeles, California 90095-1569, United States

*Corresponding authors

ana@,chem.ucla.edu

sautet@ucla.edu

\section{GAS method:}

\subsection{Flowchart of global activity search (GAS) method.}

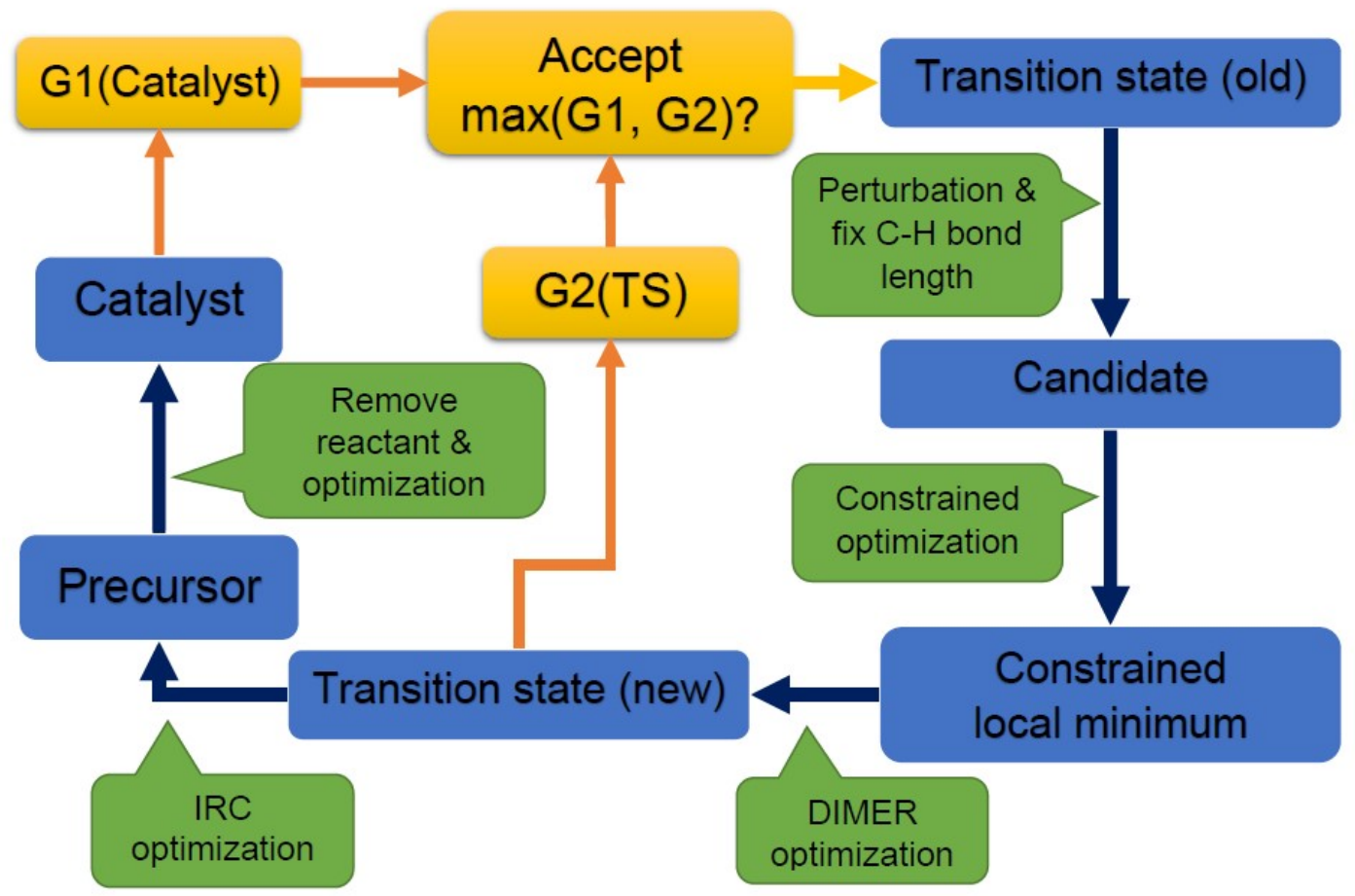

Figure S 1 Flowchart of global activity search method (GAS). 
The flowchart of the GAS is shown in Figure S 1. We have made the code publicly available at the code repository (https://github.com/GengSS/gcbh). the breakdown of each individual step is detailed as below:

(1) The sampling starts with a known TS structure for $\mathrm{CH}_{4}$ dissociation called "Transition state (old)".

(2) A new structure "Candidate" is generated from "Transition state (old)" by randomly choosing an operation including (i) adding a $\mathrm{H}$ atom, (ii) removing a $\mathrm{H}$ atom, (iii) displacing coordinates from equilibrium positions, (iv) relocating the $\mathrm{CH}_{3}-\mathrm{H}$ group from one $\mathrm{Pt}$ site to another site of the $\mathrm{Pt}_{\mathrm{n}} \mathrm{H}_{\mathrm{x}}$ cluster. To reduce the computational cost, we did not disturb the $\mathrm{d}\left(\mathrm{CH}_{3}-\mathrm{H}\right)$ (the distance of the carbon in methyl and dissociating hydrogen) in this step, and moreover, we apply a constraint to the length of $\mathrm{d}\left(\mathrm{CH}_{3}-\mathrm{H}\right)$. The $\mathrm{d}\left(\mathrm{CH}_{3}-\mathrm{H}\right)$ will change adaptively according to the structure of new $\mathrm{Pt}_{\mathrm{n}} \mathrm{H}_{\mathrm{x}}$ cluster in the following DIMER ${ }^{1}$ optimization. Initial the breaking $\mathrm{CH}_{3}-\mathrm{H}$ distance is frozen as $1.45 \AA$ though the bond length will be optimized in DIMER step accordingly.

(3) We perform a local optimization for the "Candidate" subject to the constraint on $\mathrm{d}\left(\mathrm{CH}_{3}-\mathrm{H}\right)$, and finally we get a constrained local minimum as "Constrained local minimum". This is step is conducted by BFGS method implemented in ASE (Atomic Simulation Environment Package). This step is optimized until a strict force criterion $0.01 \mathrm{eV} / \AA$.

(4) We remove the constraint on $\mathrm{d}\left(\mathrm{CH}_{3}-\mathrm{H}\right)$ and run a DIMER optimization" ${ }^{1-2}$ to get "Transition state (new)". This step is optimized by ASE package until the maximum force is bellow $0.03 \mathrm{eV} /$ Angstrom. The image separation of the DIMER images is $0.008 \AA$.

(5) (optional) We run intrinsic reaction coordination (IRC) or steepest descent optimization (with small time step) to follow the minimum mode and to obtain the precursor state ("Precursor") in which $\mathrm{CH}_{4}$ is adsorbed on a $\mathrm{Pt}_{\mathrm{n}} \mathrm{H}_{\mathrm{x}}$ isomer.

(6) (optional) We remove the $\mathrm{CH}_{4}$ and optimize the cluster, and the optimization will result in the "Catalyst", which is referred as entry cluster (EC) in the manuscript. This step is conducted by ASE package. Force is optimized until the maximum residue is below $0.03 \mathrm{eV} / \AA$.

Please note that step (5) and (6) were used to obtain the cluster configuration in the absence of $\mathrm{CH}_{4}$. In order to save computation time during GAS sampling, we simplified step (5) and (6) by directly removing $\mathrm{CH}_{4}$ group in the TS and optimizing the cluster (without $\mathrm{CH}_{4}$ ), and such a simplified practice results in the same entry cluster geometry in most cases. At the end of the GAS sampling, we double-checked these entry clusters only for those low free energy TSs by performing complete step (5) and step (6) to ensure the entry cluster is correctly assigned. Since the energy of the TS structure is the key quantity to determine the evolution of the sampling, the rare 'wrongly-assigned' entry cluster has negligible impact on the sampling, as long as its free energy is lower than the TS (shown in the Figure 2 of the main text).

(7) Calculating G2 for the TS structure of "Transition state (new)" using equation 10 of the main text and G1(free energy of the "catalyst") using equation (5) of the main text.

(8) We take the larger value between G1 and G2 as the optimization target, i.e. $G=\max (G 1, G 2)$. G2 > G1 indicates that the methane activation is the rate-determining step, and this is most common situation and responsible for all the pathways shown in Figure 2. G2 $<$ G1 indicates the cluster reconstruction is the rate-determining step, and those cases only occur for high energy metastable isomers. Finally, the standard Metropolis algorithm ${ }^{3}$ is used to accept new structures, i.e.

$$
p=\min \left[1.0, \exp \left(-\frac{G(\text { new })-G(\text { old })}{k_{B} T}\right)\right]
$$


In equation (1), $k_{B}$ is the Boltzmann constant and $T=2000 \mathrm{~K}$ is a parameter. If $G(n e w)$ is lower than $G($ old $)$, the new structure is always accepted. Otherwise, the new structure is accepted with a probability defined in the equation (1).

Please note that the energy of the barrier $\left(E_{T S}\right)$ shown in Figure 2 is defined as $E_{T S}=G(T S)-G(G M)$, in which $G(G M)$ is the free energy of the global minimum (for clusters). Because $G(G M)$ is a constant at chosen condition, the minimizing target $\mathrm{G} 2$ is essentially equivalent to minimize $E_{T S}$.

The original Basin-Hopping method is serial; therefore, the demonstrated flowchart of GAS is found to be slow in the sampling. Two strategies can be used to improve the time efficiency. 1) Carrying out many GAS instances in parallel, each of them being standalone without communicating with others; 2) Using the "pool" method. In this case, the paralleled GAS instances will combine their results into a single 'pool' and if any instance finds that the 'pool' contains better solutions (TS with lower free energy) than it currently holds, it will abandon its own solution and accept the solution in the pool as starting point for next loop. Finally, the reported results are the outcome of two stages, we use method 1 at the beginning for parallelizing GAS first, but we found that some GAS instances are stuck at high energy TSs compared with others. In the second stage, we resume the sampling with the pool-version GAS to extended the sampling efficiency.

\subsection{Total number of force/energy evaluations}

In the case of $\mathrm{Pt}_{8}\left(\mathrm{Pt}_{7}\right)$ cluster, we have conducted 614 (916 for $\left.\mathrm{Pt}_{7}\right)$ GAS loops and each of them consists in a constrained local optimization, a DIMER optimization and one precursor optimization. Totally, we consumed 178156 (308713 or $\mathrm{Pt}_{7}$ ) force/energy calls (in average 285 (337 for $\mathrm{Pt}_{7}$ ) per loop). The constrained local optimization in average uses 184 (212 for $\left.\mathrm{Pt}_{7}\right)$ force/energy calls per loop since it starts from disturbed structures. DIMER optimization uses 27 (54 for $\mathrm{Pt}_{7}$ ) force/energy calls, thanks to the good initial guess provided by constrained local optimization. The final precursor optimization uses 74 ( 70 for $\mathrm{Pt}_{7}$ ) force/energy calls per loop. Note that $\mathrm{Pt}_{7}$ uses more CPU time because the sampling of $\mathrm{Pt}_{7}$ starts with zero knowledge on the structure of the stable pure clusters on alumina, while for $\mathrm{Pt}_{8}$ we knew the GM of the supported cluster from previous research. 


\subsection{Slab model}
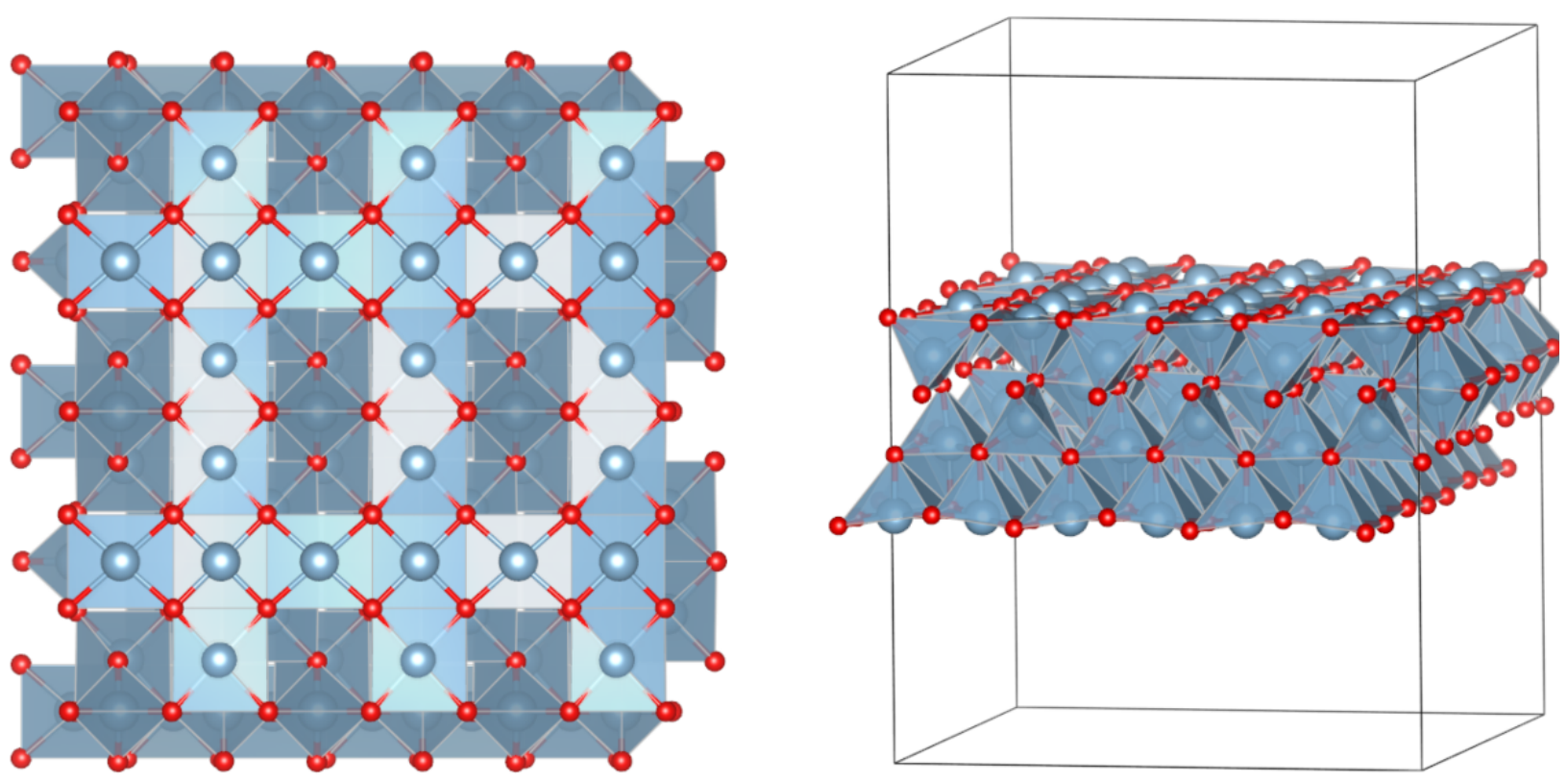

Figure S 2: the slab mode used in this manuscript. Top view (left) and side view (right)

\section{Structures of low-free energy clusters}

The GAS method itself generates the pairs of entry clusters (ECs) and transition states (TSs), and lowers the free energy of both simultaneously. Therefore, one does not need to explore the clusters with a separate method before. While, considering the GAS method is more expensive than standard global optimizations. One can use the results of the low-lying structures as initial guess to accelerate the GAS sampling. Therefore, we explored the low free energy metastable ensemble of configurations for each supported cluster under the same condition by conventional grand canonical genetic algorithm runs ${ }^{4}$. Altogether, 2506 (resp. 1773) distinct structures are sampled for $\mathrm{Pt}_{7} \mathrm{H}_{\mathrm{x}}$ (resp. $\mathrm{Pt}_{8} \mathrm{H}_{\mathrm{x}}$ ) on $\gamma-\mathrm{Al}_{2} \mathrm{O}_{3}$ under 0.1 bar of $\mathrm{H}_{2}$ at $600^{\circ} \mathrm{C}$ and the ensemble of low free energy metastable configurations is shown in Figure $\mathrm{S} 3$. Both the cluster geometry and its hydrogen coverage are free to change, the hydrogen chemical potential being fixed by the conditions. 18 unique cluster configurations are found for both $\mathrm{Pt}_{7}$ and $\mathrm{Pt}_{8}$ cluster in the free energy window of $0.3 \mathrm{eV}$ above the GMs, and they present relative populations bigger than $1 \%$ estimated by the Boltzmann distribution at $T=600^{\circ} \mathrm{C}$ (Figure $\mathrm{S} 3$ (a), (b)). These clusters show similar hydrogen coverage ranging from 4 to $6 \mathrm{H}$ atoms. The GMs of $\mathrm{Pt}_{7} \mathrm{H}_{\mathrm{x}}$ and $\mathrm{Pt}_{8} \mathrm{H}_{\mathrm{y}}$ are shown in Figure $\mathrm{S} 3$ (c) and Figure $\mathrm{S} 3$ (d), respectively; both bear 6 hydrogen atoms. The $\mathrm{GM}$ of $\mathrm{Pt}_{8} \mathrm{H}_{6}$ is flat on the $\gamma-\mathrm{Al}_{2} \mathrm{O}_{3}(100)$ surface, but for $\mathrm{Pt}_{7} \mathrm{H}_{6}$, one $\mathrm{Pt}$ atom is detached from the surface. Structures of other low free energy clusters are shown in Figure S4 and S5. 

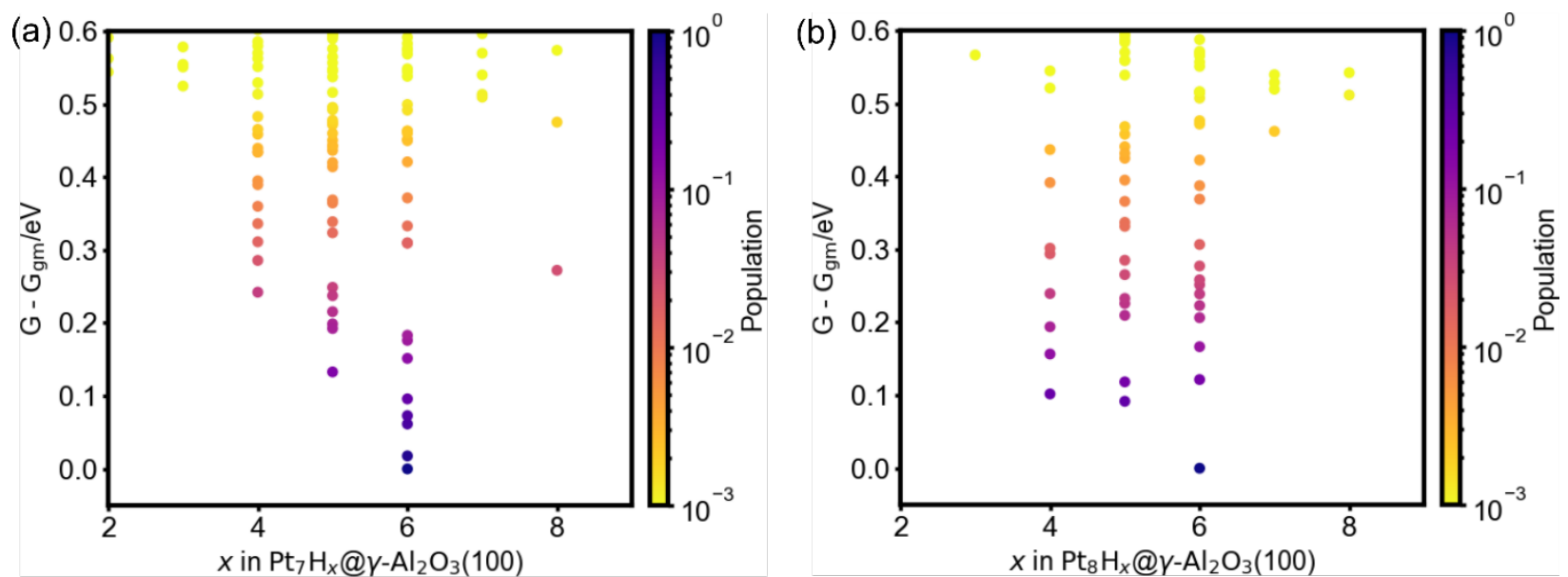

(c)

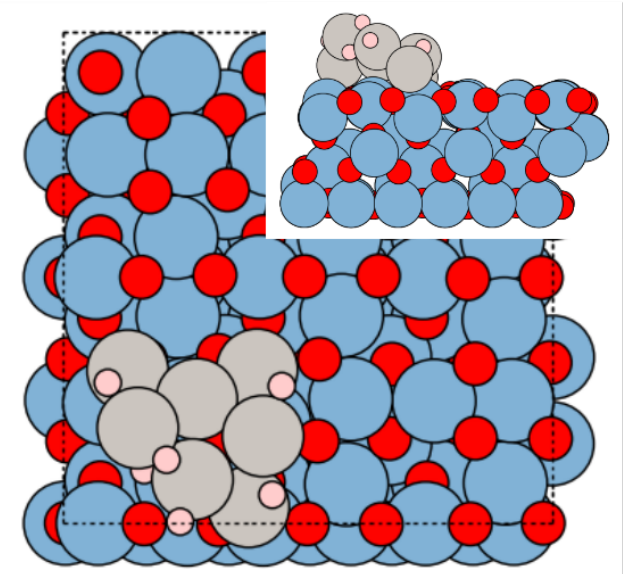

(d)

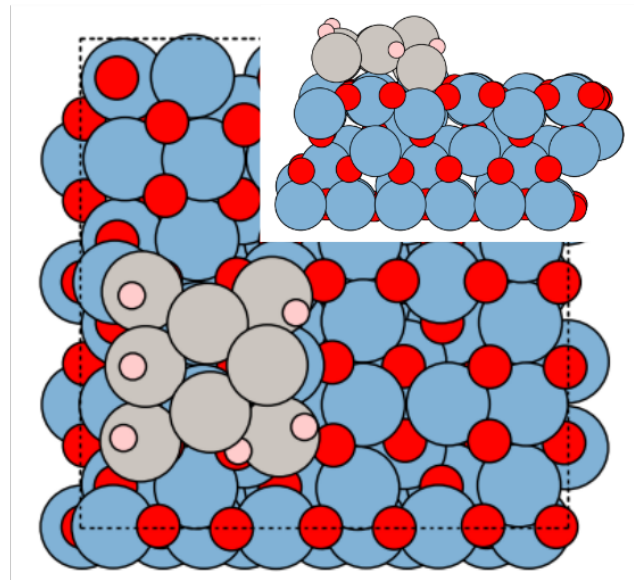

Figure $S 3$ The low energy ensemble of configurations (GM to GM+0.3 eV) for $\mathrm{Pt}_{7} H_{x}(a)$ and $\mathrm{Pt}_{8} \mathrm{H}_{x}(b)$ clusters supported on $\gamma-\mathrm{Al}_{2} \mathrm{O}_{3}(100)$, characterized by their number of $\mathrm{H}$ atoms ( $x$ axis) and their reconstruction free energy ( $y$ axis). The relative population of the different configurations is coded by the color of the dot. The conditions correspond to a temperature of $600{ }^{\circ} \mathrm{C}$ and a pressure of $\mathrm{H}_{2}$ of 0.1 bar. (c) and (d) show the most stable structure for the $\mathrm{Pt}_{7} \mathrm{H}_{x}$ cluster and $\mathrm{Pt}_{8} \mathrm{H}_{x}$ cluster, with the side view inserted. The optimal coverage is 6 hydrogen atoms for both clusters. 

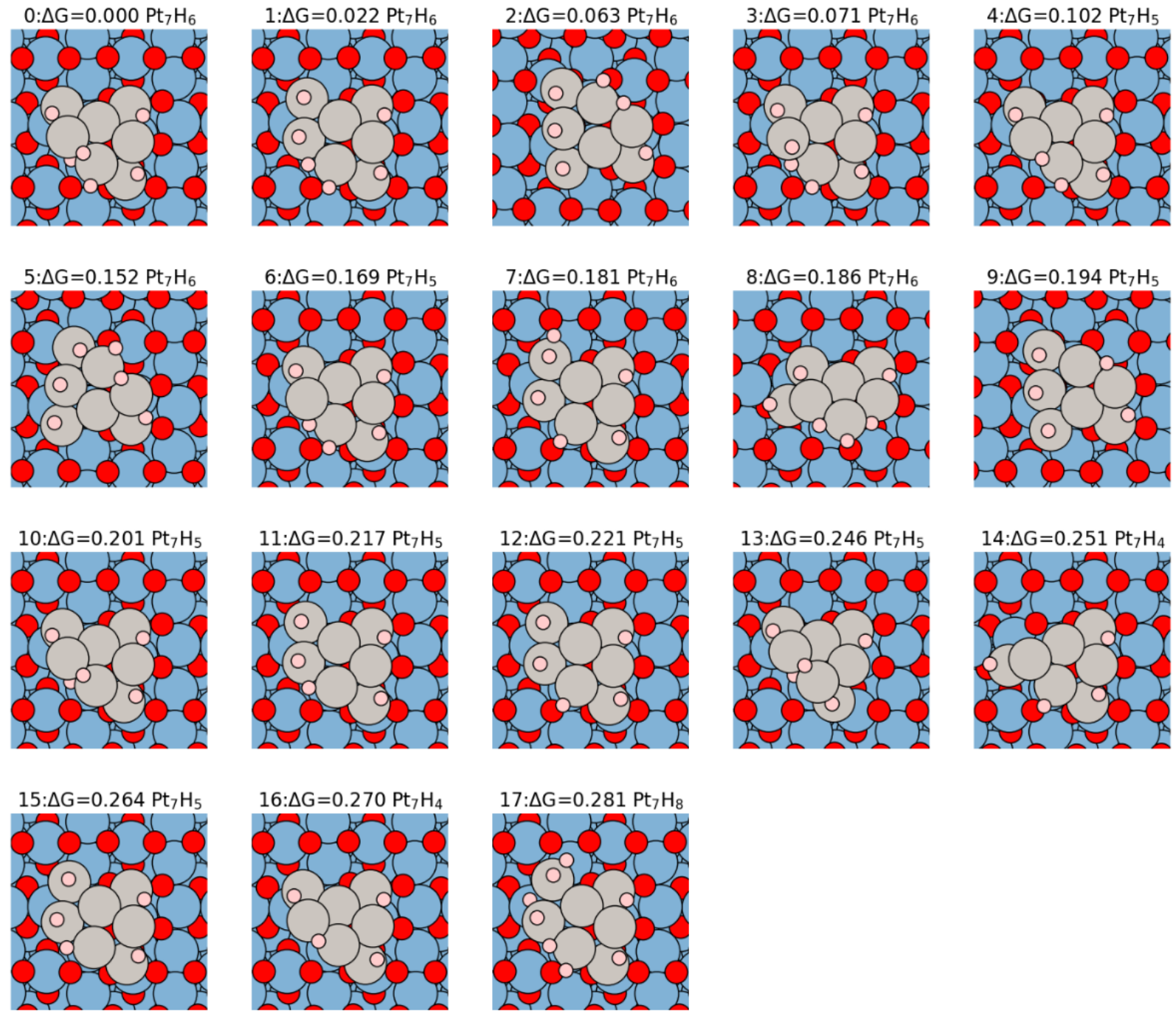

Figure $S 418$ unique low free energy structures for $\mathrm{Pt}_{7}$ on $\gamma-\mathrm{Al}_{2} \mathrm{O}_{3}(100)$ in the free energy gap of GM to $G M+0.3 \mathrm{eV}$. The relative free energies, and chemical formula of the cluster (excluding support) are shown in the top of each structure in eV unit. 

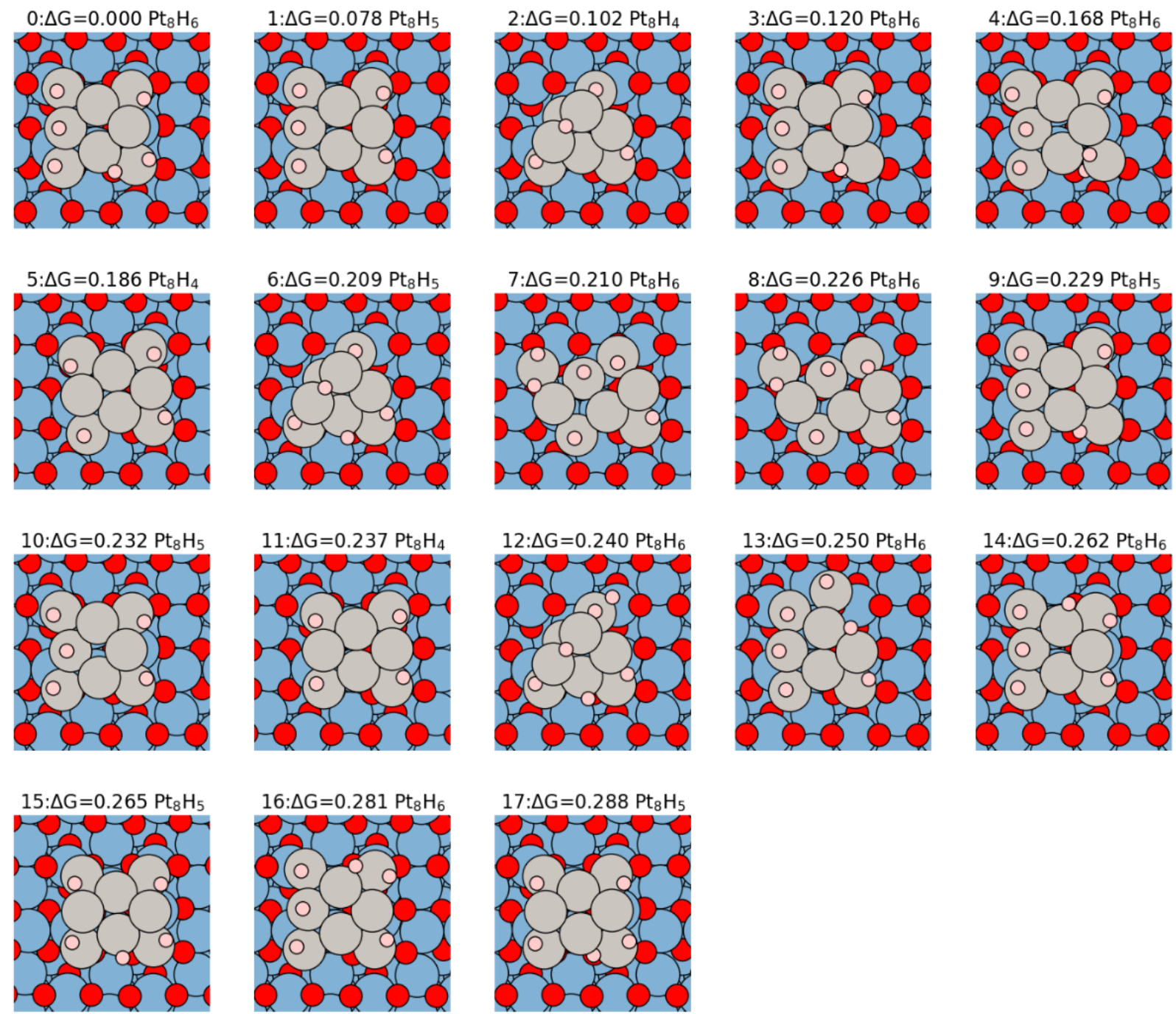

Figure $S 518$ unique structures for Pt $t_{8}$ clusters on $\gamma-\mathrm{Al}_{2} \mathrm{O}_{3}(100)$ in the free energy window from GM to $G M+0.3 \mathrm{eV}$. The relative free energies, and chemical formula of the cluster (excluding support) are shown in the top of each structure $\mathrm{eV}$ unit. The ensemble of local minima structures for $\mathrm{Pt}_{8} \mathrm{H}_{x}$ are extended from a previous paper $\left[{ }^{5}\right]$, and some of them are replotted here. 


\section{Structure reconstruction along the activation}

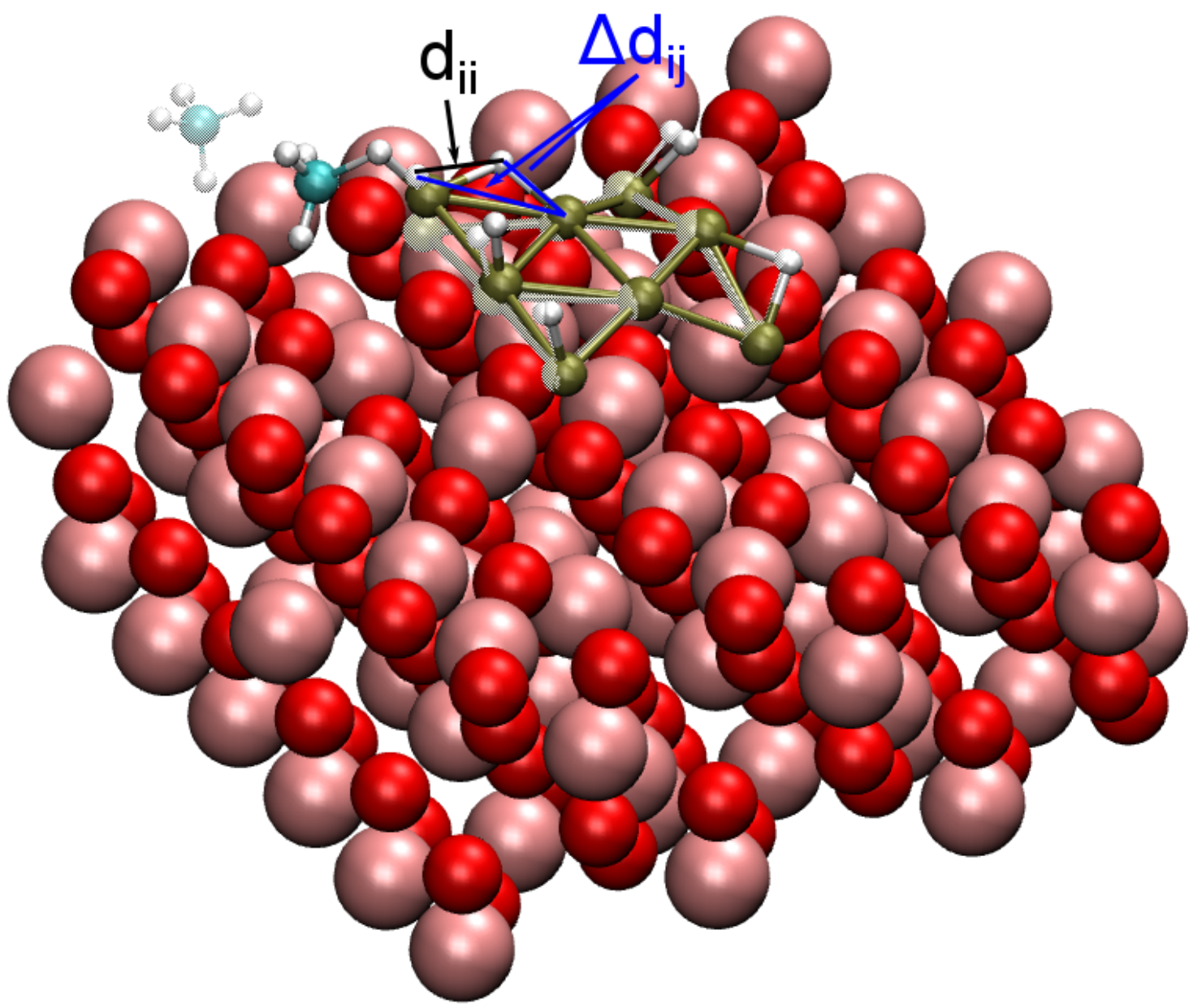

Figure $S 6$ The definition of $d_{i i}$ and $d_{i j}^{r e f}$. The TS structure is shown together with the initial structure (IS) (gas phase $\mathrm{CH}_{4}$ and cluster are shown as semi-transparent style). $d_{i i}$ is the displacement of the same atom from IS to TS showing how much an atom from cluster translates during the activation. Therefore, $d_{i j}$ has the unit of Angstrom. $d_{i j}^{\text {ref }}$ shows the change of interatomic distance between atoms $i$ and $j$ from the IS to the TS, i.e. $d_{i j}^{r e f}=\left|d_{i j}(T S)-d_{i j}(I S)\right| . d_{i j}^{\text {ref }}$ is reported normalized by the sum of covalent radius, i.e. $d_{i j}^{r e f}=d_{i j}^{r e f} /\left(r_{i}^{0}+r_{j}^{0}\right)$, in which $r_{i}^{0}$ and $r_{j}^{0}$ are the covalent radius of atom $i$ and $j$ respectively. In practice when evaluating $d_{i j}^{\text {ref }}$, we also neglected the situation that $d_{i j}(T S)>1.5 \times\left(r_{i}^{0}+\right.$ $\left.r_{j}^{0}\right)$ and $d_{i j}(I S)>1.5 \times\left(r_{i}^{0}+r_{j}^{0}\right)$, or $d_{i j}(T S)<\left(r_{i}^{0}+r_{j}^{0}\right)$ and $d_{i j}(I S)<\left(r_{i}^{0}+r_{j}^{0}\right)$. Finally, if $d_{i j}^{r e f}>$ 0.5 , we can say that bond between atom $i$ and $j$, is broken or formed during the activation. 

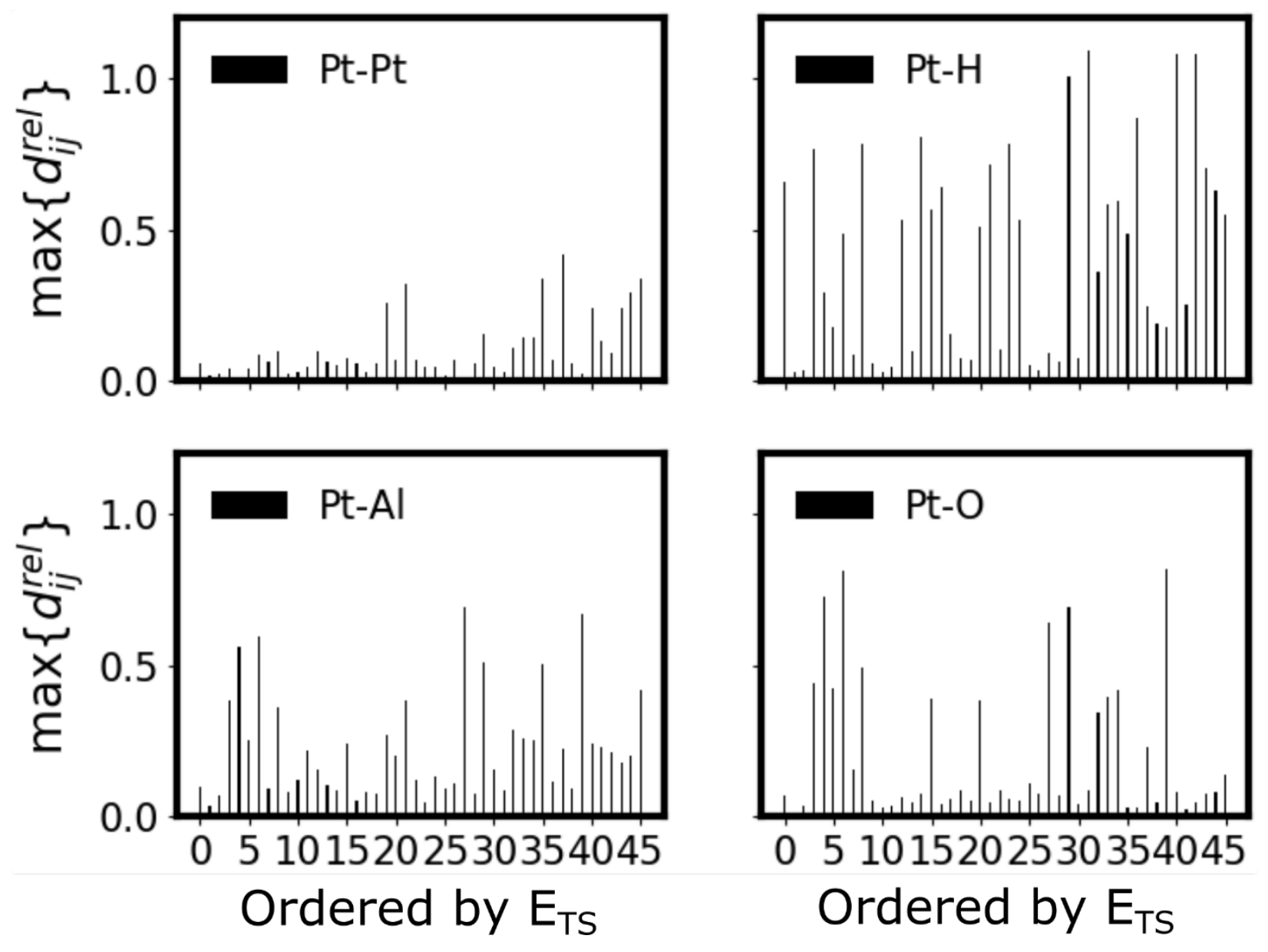

Figure $S$ 7: For $\mathrm{Pt}_{7}$ on $\gamma-\mathrm{Al}_{2} \mathrm{O}_{3}$, and for each reaction channel (ordered by increasing $E_{T S}$ value), the maximum $d_{i j}^{r e l}$ values for Pt-Pt, Pt-H, Pt-Al and Pt-O pairs are shown. 

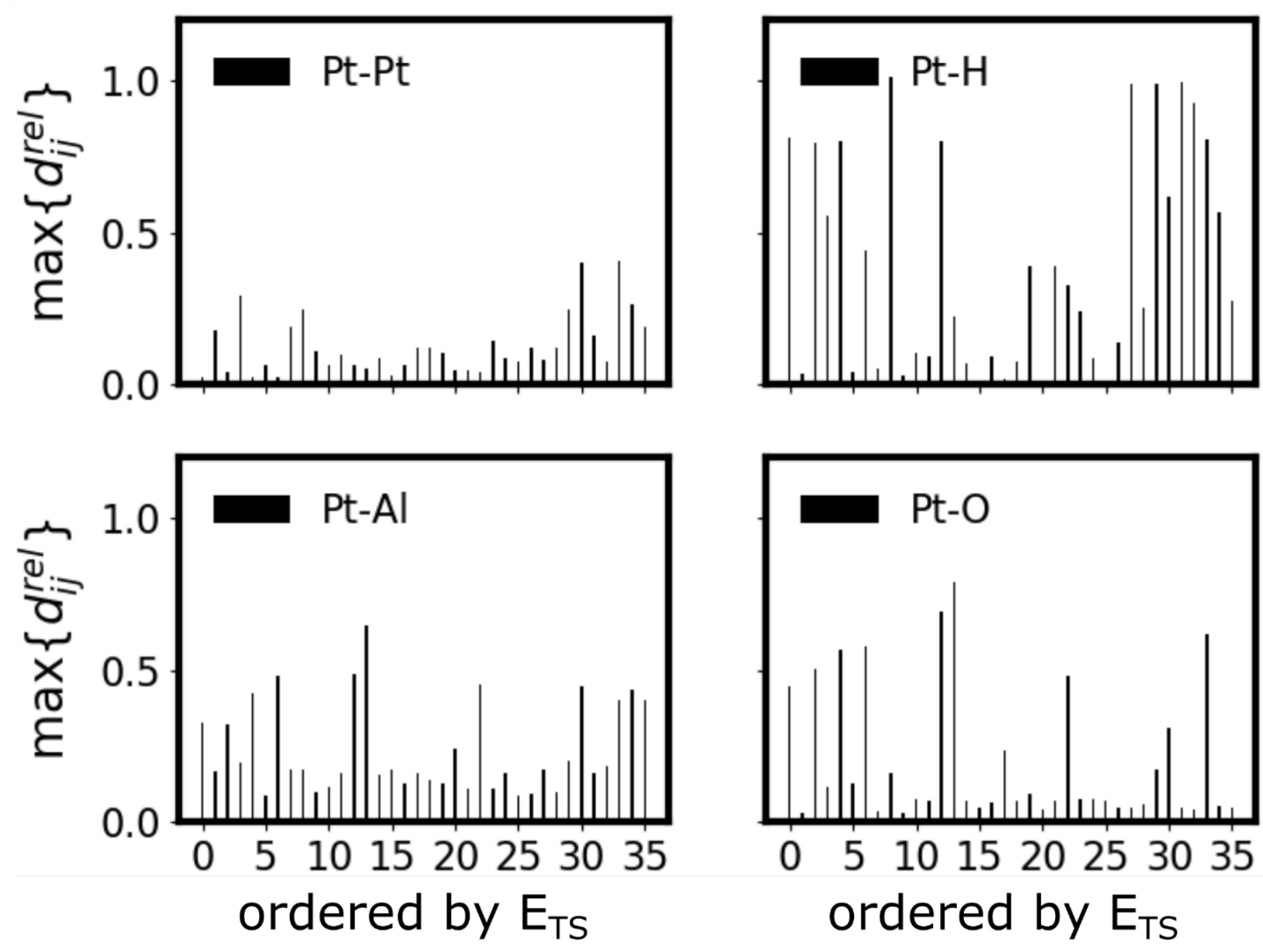

Figure $\mathrm{S} 8 \mathrm{For} \mathrm{Pt}_{8}$ on $\gamma-\mathrm{Al}_{2} \mathrm{O}_{3}$, and for each reaction channel (ordered by increasing $E_{T S}$ value), the maximum $d_{i j}^{r e l}$ values for Pt-Pt, Pt-H, Pt-Al and Pt-O pairs are shown. 

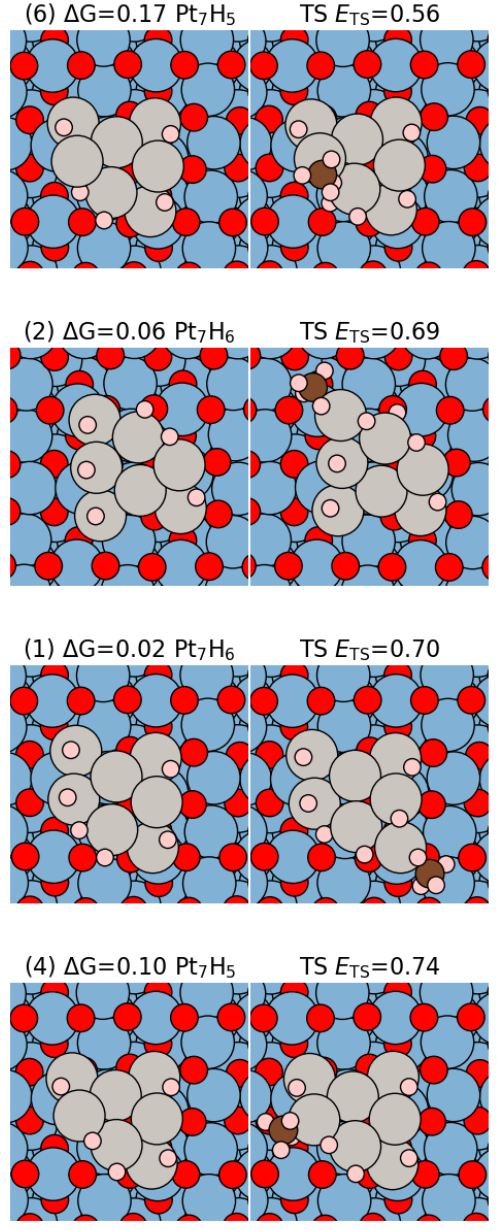
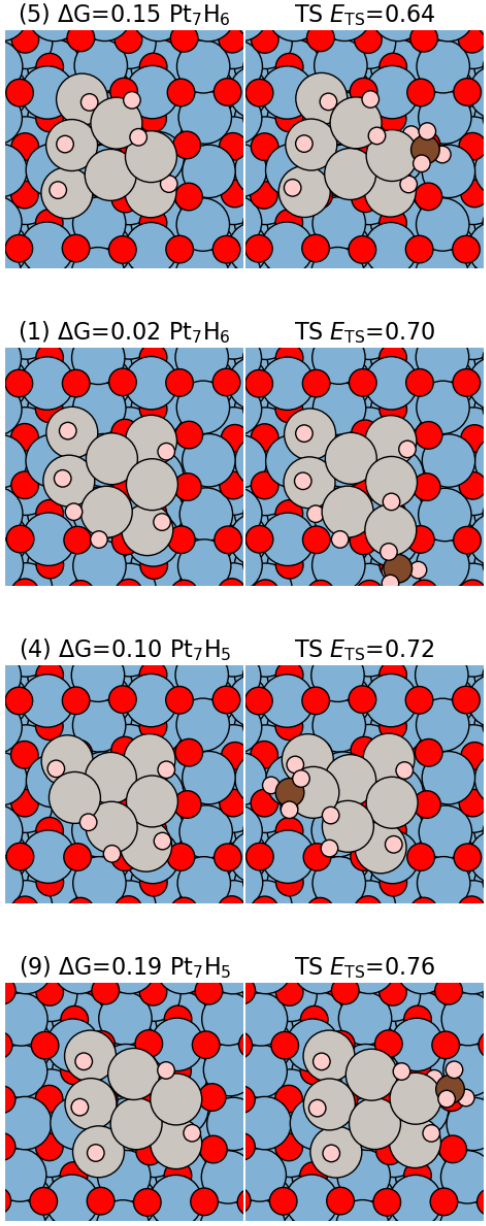

(4) $\Delta \mathrm{G}=0.10 \mathrm{Pt}_{7} \mathrm{H}_{5} \quad \mathrm{TS} E_{\mathrm{TS}}=0.66$

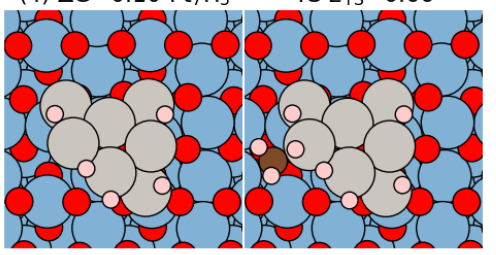

(8) $\Delta \mathrm{G}=0.19 \mathrm{Pt}_{7} \mathrm{H}_{6} \quad \mathrm{TS} E_{\mathrm{TS}}=0.70$

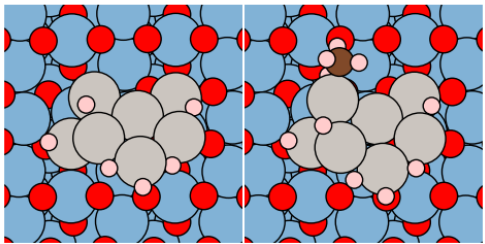

(9) $\Delta \mathrm{G}=0.19 \mathrm{Pt}_{7} \mathrm{H}_{5} \quad \mathrm{TS} E_{\mathrm{TS}}=0.72$

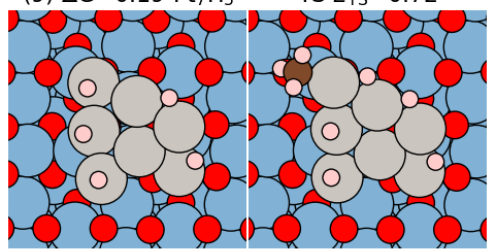

(9) $\Delta \mathrm{G}=0.19 \mathrm{Pt}_{7} \mathrm{H}_{5} \quad \mathrm{TS} E_{\mathrm{TS}}=0.77$

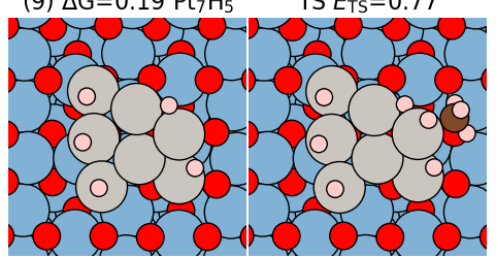

Figure $S 9$ The first 12 most competitive reaction channels for the Pt $t_{7}$ cluster on $\gamma-\mathrm{Al}_{2} \mathrm{O}_{3}(100)$. Each panel consists of two images, left is the entry cluster configuration and the right is the TS. On top of each cluster configuration, the relative stabilities, and chemical formula of the cluster are shown. On top of each TS, the $E_{T S}$ and chemical formula of the cluster are also shown. Unit is eV for all numbers. 

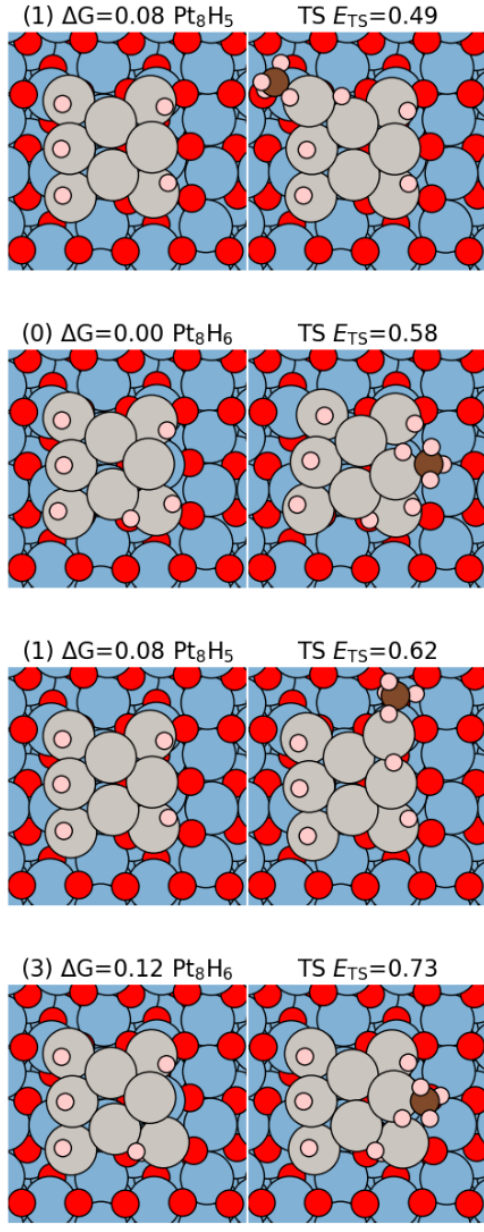
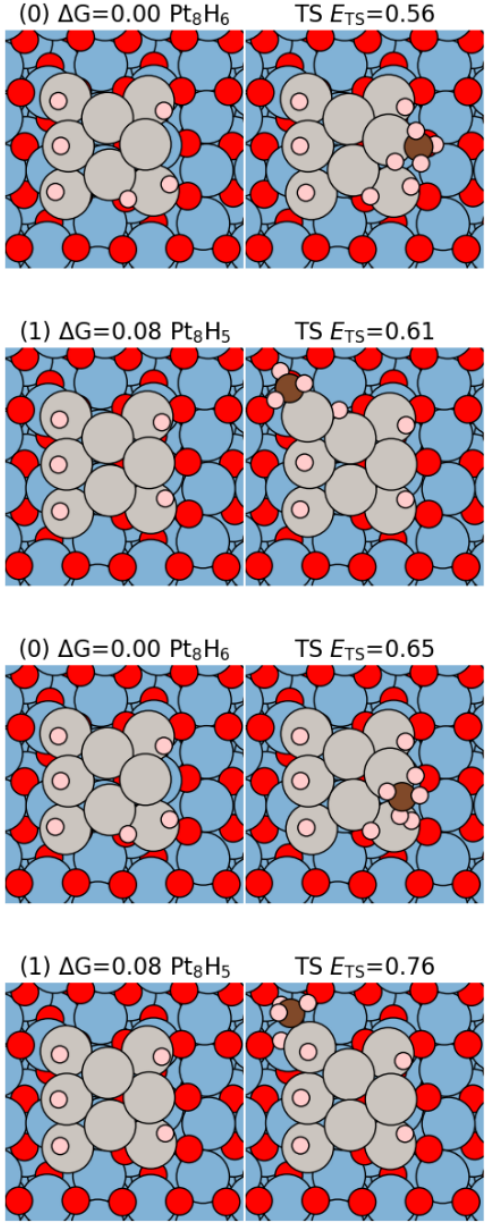
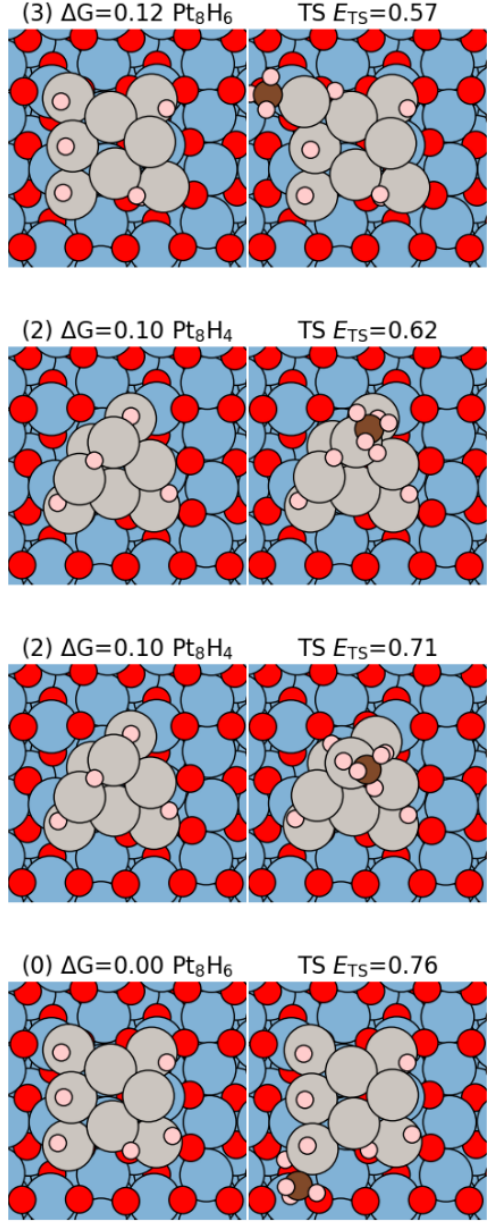

Figure S 10 The first 12 most competitive reaction channels on Pt $t_{8}$ cluster. Each panel consists of two images, left is the cluster configuration and the right one is the TS. On the top of each cluster configuration, the relative stabilities, and chemical formula of the cluster is shown. On the top of each TS, the $E_{T S}$ and chemical formula of the cluster are also shown. Unit is eV for all numbers. The ensemble of local minima structures for $\mathrm{Pt}_{8} \mathrm{H}_{x}$ are extended from a previous paper $\left[{ }^{5}\right]$, and some of them are replotted here. 


\section{Manual exploration of TS structures}

A manual approach was conducted for exploring the transition states (TSs) from a metastable configuration $\left(\mathrm{Pt}_{8} \mathrm{H}_{5}\right)$ which provides the optimal reaction channel on $\mathrm{Pt}_{8}$ cluster (the one providing the lowest $\mathrm{E}_{\mathrm{TS}}$ shown in Figure 2). The results of manual explorations are shown in Figure S 11. We explored 14 different cases, and the optimization procedure for obtaining the TSs is:

(1) First, a methane with elongated $\mathrm{C}-\mathrm{H}$ bond (1.525 $\AA$ is used in this case) is intentionally placed on the $\mathrm{Pt}_{8} \mathrm{H}_{5}$ cluster. We explored two types of Pt sites: Figure S 11(1-11) explore the vacant sites that are free of $\mathrm{H}$ atoms, and Figure S 11(12-14) explore the H occupied Pt sites which is identified as the optimal site for methane activation from the sampling. We also examined several arrangements of the activated methane on the selected Pt sites. In all the cases, we did not disturb the geometry of the cluster after attaching the methane. This is consistent with standard procedure since we do not know the optimal way to deform the cluster in the coordinate space of dimension 39 (13 atoms $\times 3$ directions).

(2) Second, the structures are optimized while freezing the length of the elongated $\mathrm{C}-\mathrm{H}$ bond, and then they are further refined with DIMER method afterwards removing the constraint on the $\mathrm{C}-\mathrm{H}$ bond.

Finally, we obtained two types of results: The DIMER optimizations in Figure S 11(1-8) end up with high energy gas-phase methyl radicals which are not valid TSs, hence only the constrained local minima are shown. Initial structures in Figure S 11(9-14) generated valid TS structures. In all the cases, we showed the $\Delta E_{a}=G\left(T S_{\text {current }}\right)-G\left(T S_{\text {optimal }}^{\text {sampling }}\right)$ to indicate the energy difference between this manual approach and the fully sampled results.

The TS structures shown in Figure S 11(9-14) are at least $0.26 \mathrm{eV}$ higher than the optimal TS found in the sampling. Meanwhile, we found that those vacant Pt sites, which seem to be potential active sites at first sight, are almost inert (shown in Figure S 11(9-11)) or unable to catalyze methane activation at all (Figure S 11(1-8)). Figure S 11(12-14) show three cases starting from the optimal Pt site (insights gained from sampling), and those sites are apparently more active than others though they are more saturated. Apparently, the current manual approach only finds TSs with a configuration of the cluster close to the initial guess and misses the deformation of the cluster (lifting of Pt and migrating of $\mathrm{H}$ ) found by the sampling method. Therefore, manual approach overestimates the barrier by $0.26 \mathrm{eV}$. 

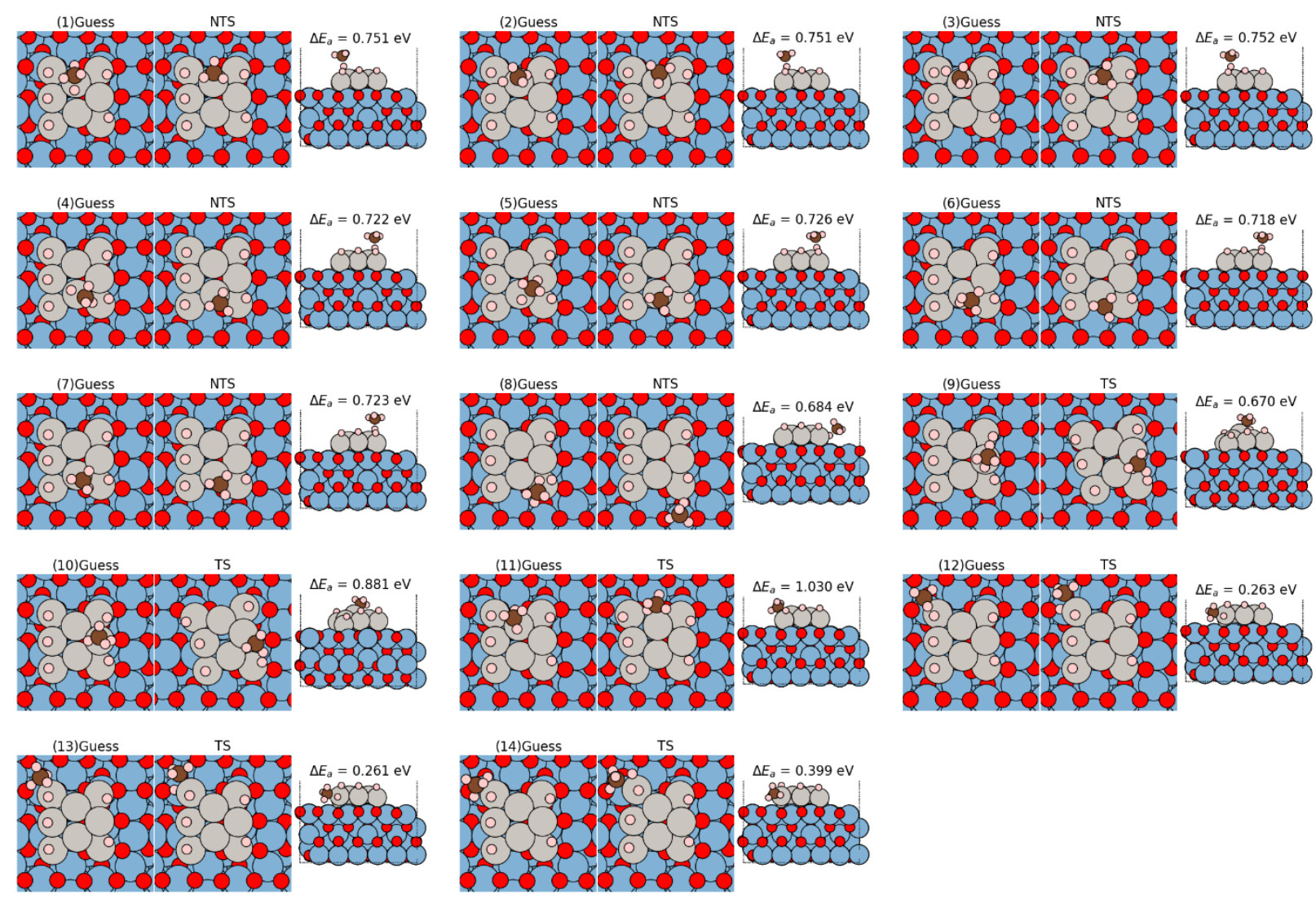

Figure $S 11$ Manually exploring the TSs from a metastable configuration of $\mathrm{Pt}_{8}\left(\mathrm{Pt}_{8} \mathrm{H}_{5}\right)$. Each threecolumn panel shows a case of manual exploration. The first panel shows a methane with elongated $C-H$ bond (1.525 A) intentionally placed on the Pt site, the second panel shows the obtained TS (or constrained local minima in 1-8), and the third panel shows the side view of the obtained TSs (or constrained local minima in 1-8). NTS indicates "Not TS". $\Delta E_{a}$ indicates the energy difference between the current transition states and the optimal one found in the sampling (the one with lowest $\left.E_{T S}\right)$ ). 


\section{A shallow intermediate state for $\mathrm{C}-\mathrm{H}$ bond breaking.}

In Figure 2 of the manuscript, the TSs structures are obtained firstly by DIMER method, and then the entry cluster is obtained by steepest descent (SD) optimization from a slightly displaced geometry of the TS (the displacements are applied along the virtual mode). The SD trajectory mimics the minimum mode path from the TS to a local minimum in the vicinity of the TS. Considering the weak interaction between the cluster and methane, most final products of SD optimizations are entry clusters and gas phase $\mathrm{CH}_{4}$ molecules. However, not all the cases will trace back to the exact geometry of the entry cluster after SD optimization, instead some of them (12 out of 46 pathways for $\mathrm{Pt}_{7}$ cluster, and 8 of 36 pathways for $\mathrm{Pt}_{8}$ ) will result in precursor states in which the methane molecules are slightly activated with short $\mathrm{C}-\mathrm{H}$ bonds $(\mathrm{d}<1.15)$ binding with the cluster, and the clusters are in the deformed geometries which are similar to the TSs rather than the entry clusters. Such a precursor state has to undergo a small barrier to release the methane molecules to the gas phase (Figure S 12) and retain the geometry of the entry cluster itself. An example, which is the optimal reaction channel on $\mathrm{Pt}_{8}$ and consists of such a precursor state, is shown in Figure S 13.
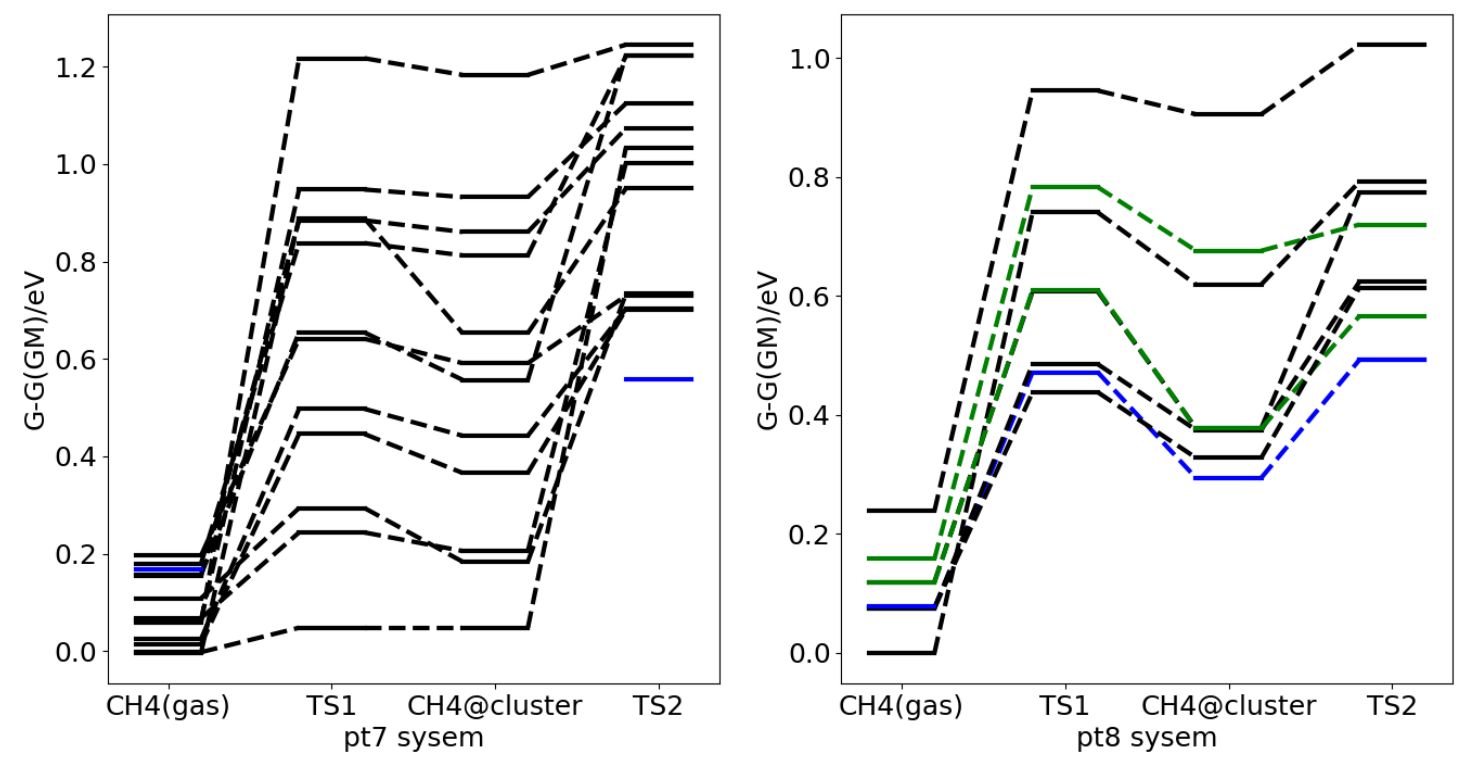

Figure S 1212 out of 46 pathways for Pt $t_{7}$ and 8 out of 36 pathways for Pt $t_{8}$ (in Figure 2 of the manuscript) consist of a shallow intermediate state when the gas phase $\mathrm{CH}_{4}$ approaches to the TS2 in which the C-H breaking occurs. Left panel shows the profiles for Pt $t_{7}$ cluster and right panel shows the profiles for Pt $t_{8}$ cluster for which there exists an intermediate state. Structures of a typical example (blue curve in the right panel, being the optimal pathway for Pt $t_{8}$ system) are shown in Figure S 13. $\mathrm{CH}_{4}$ (gas) indicates the state of $\mathrm{CH}_{4}(\mathrm{gas})$ plus entry cluster (e.g. Figure S 13(a)), TS1 (e.g. Figure S 13(b))indicates the transition state structure to form the intermediate state; $\mathrm{CH}_{4} @$ cluster indicates the intermediate state(e.g. Figure S 13(c)), and finally, the TS2 indicates the transition state of C-H breaking for methane dissociation found in the GAS sampling. The two blue profiles show the optimal reaction pathways for Pt ${ }_{7}$ and $\mathrm{Pt}_{8}$, respectively, in which the optimal pathway for $\mathrm{Pt}_{7}$ does not contain an intermediate structure, but the one for Pt $t_{8}$ does. The green curves show the only two cases that the TS1 is higher than TS2 in energy (by around $0.05 \mathrm{eV}$, see text of the SI below).

The transition states to form the intermediate states (e.g. TS1 in Figure S 12 and Figure S 13) are lower in energy compared with the transition states to form dissociated species (e.g. TS2 in Figure S 12 or Figure S 13) for most cases. The only exceptions are the two cases which are shown with the green profiles in 
Pt8 system (Figure S 12), in which the TS1 is higher than the TS2 by 0.04 and $0.06 \mathrm{eV}$, respectively. However, as seen in the manuscript of Figure 3(d), those two green pathways contribute only the minor part of the global rate (only the first two isomers of $\mathrm{Pt}_{8}$ are important). Hence, the presence of those intermediate states does not impact our conclusion in the main text.

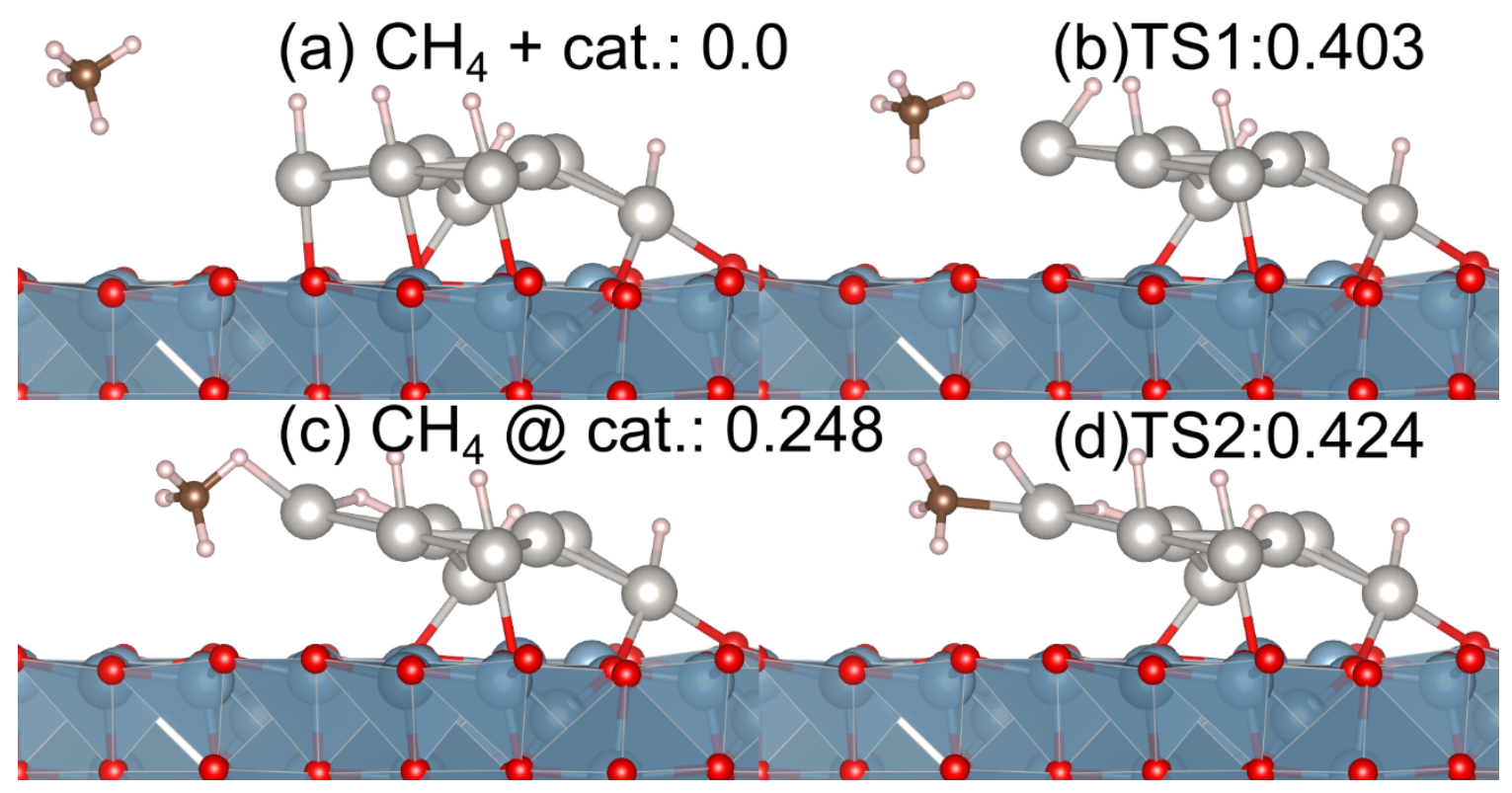

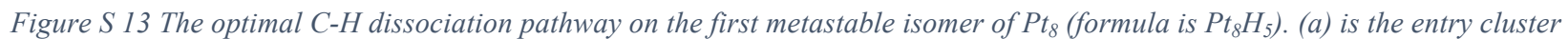
plus a gas-phase methane molecule; (b) is the TS to form the intermediate state (c); (d) is the rate-determining transition state (found in GAS method) to form the $\mathrm{C}$-H dissociated product; The numbers show the relative stability of each structure in unit eV, and the reference is the energy of the cluster isomer (i.e. structure (a)). 


\section{References}

1. Henkelman, G.; Jónsson, H., A Dimer Method for Finding Saddle Points on High Dimensional Potential Surfaces Using Only First Derivatives. J. Chem. Phys. 1999, 111, 7010-7022.

2. Melander, M.; Laasonen, K.; Jonsson, H., Removing External Degrees of Freedom from Transition-State Search Methods Using Quaternions. J Chem Theory Comput 2015, 11, 1055-62.

3. Wales, D. J.; Doye, J. P. K., Global Optimization by Basin-Hopping and the Lowest Energy Structures of Lennard-Jones Clusters Containing up to 110 Atoms. J. Phys. Chem. A 1997, 101, 51115116.

4. Sun, G.; Alexandrova, A. N.; Sautet, P., Pt8 Cluster on Alumina under a Pressure of Hydrogen: Support-Dependent Reconstruction from First-Principles Global Optimization. J. Chem. Phys. 2019, 151, 194703.

5. Sun, G.; Sautet, P.; Alexandrova, A. N., Pt8 Cluster on Alumina under a Pressure of Hydrogen: Support-Dependent Reconstruction from First-Principles Global Optimization J. Chem. Phys. 2019, accepted. 